\title{
BMJ Open KETOgenic diet therapy in patients with HEPatocellular adenoma: study protocol of a matched interventional cohort study
}

\author{
Christiaan Albert Johan Oudmaijer (D) ,, ${ }^{1,2}$ Kirsten Anna Berk, ${ }^{3}$ \\ Elisabeth Johanna Theresia Maria van der Louw, ${ }^{3}$ Rob de Man, ${ }^{4}$ \\ Aart-Jan van der Lelij, ${ }^{5}$ Jan Hendrik Jozef Hoeijmakers, ${ }^{2,6}$ Jan IJzermans ${ }^{1}$
}

To cite: Oudmaijer CAJ, Berk KA, van der Louw EJTM, et al. KETOgenic diet therapy in patients with HEPatocellular adenoma: study protocol of a matched interventional cohort study. BMJ Open 2022;12:e053559. doi:10.1136/ bmjopen-2021-053559

- Prepublication history and additional supplemental material for this paper are available online. To view these files, please visit the journal online (http://dx.doi.org/10.1136/ bmjopen-2021-053559).

Received 20 May 2021 Accepted 19 January 2022

A) Check for updates

(c) Author(s) (or their employer(s)) 2022. Re-use permitted under CC BY-NC. No commercial re-use. See rights and permissions. Published by BMJ.

For numbered affiliations see end of article.

Correspondence to Christiaan Albert Johan Oudmaijer;

c.oudmaijer@erasmusmc.nl

\section{ABSTRACT}

Introduction Hepatocellular adenoma (HCA) is an uncommon, solid and benign liver lesion, mainly occurring in women using oral contraceptives. Patients are advised to stop using oral contraceptives (OC) and, as overweight is frequently observed, dietary restrictions. Metabolic changes are assumed to play a role and it has been suggested that diet may help to reduce tumour size. A low-calorie ketogenic diet (LCKD) has been shown to induce weight loss and multiple metabolic changes, including the reduction of portal insulin concentrations, which downregulates hepatic growth hormone receptors. Weight reduction and an LCKD can potentially reduce the size of HCAs.

Methods and analysis We designed a matched, interventional cohort study to determine the effect of an LCKD on the regression of HCA. The study population consists of female subjects with an HCA, 18-50 years of age, body mass index $>25 \mathrm{~kg} / \mathrm{m}^{2}$, who are entering a surveillance period including cessation of $\mathrm{OC}$. A historical control group will be matched. The intervention consists of an LCKD (approximately $35 \mathrm{~g}$ carbohydrate $/ 1500 \mathrm{kcal} /$ day) for 3 months, followed by a less strict LCKD for 3 months (approximately $60 \mathrm{~g}$ carbohydrate $/ 1500 \mathrm{kcal} /$ day). Main study endpoint is the diameter of the HCA after 6 months, as compared with the historic control group. Secondary endpoints include adherence, quality of life, change in physical activity, liver fat content, body weight, body composition and resting energy expenditure.

Ethics and dissemination The medical ethical committee has approved the study protocol, patient information files and consent procedure and other study-related documents and procedures.

Trial registration number NL75014.078.20; Pre-results. https://www.trialregister.nl/trial/9092

\section{INTRODUCTION}

Hepatocellular adenoma (HCA) is an uncommon, solid, benign liver lesion. ${ }^{1-5}$ Typically, it is a solitary lesion found in young women within their reproductive years. HCA has been widely associated with the use of oestrogen-containing medication. The
Strengths and limitations of this study

- Study performed in a university hospital with experts on liver tumours and endocrinology.

- Availability of a large cohort of prospectively registered patients with adenoma allowing proper matching.

- Extensive statistical analysis with reduction of residual confounding to determine treatment effect.

- A large and highly motivated population of patients with hepatocellular adenoma.

Intervention is at risk for unforeseen non-adherence.

annual incidence of HCA has been estimated to be 30-40 cases per million oral contraceptive (OC) users in comparison with one case per million non-users. ${ }^{12}$ Studies have shown a consistent effect of sex hormones: the 30-40-fold increase has been confirmed in long-term OC users. ${ }^{167}$ A dose-related risk ratio and regression after cancellation of use of contraceptive pills further confirmed the effect of sex hormones in the development of HCA. ${ }^{4} 589$ More recently, studies have shown a rising incidence of diagnosis with HCA. ${ }^{10-13}$ Current prevalence is estimated to be between $0.001 \%$ and $0.004 \%$ with a reported female: male ratio of 10:1. ${ }^{14} 15$ This increasing incidence may be attributed to an increased discovery of asymptomatic HCA by extensive use of imaging, but the rising incidence of obesity has also been postulated as an influencing factor. ${ }^{10-12} 16$ In men, the use of anabolic steroid hormones (AS) may play a role. ${ }^{10}$

HCA is diagnosed by typical findings on contrast-enhanced, cross-sectional imaging by MRI. ${ }^{14}$ 17-19 A biopsy is not indicated for female patients when worrying signs are absent. ${ }^{14}$ 17-19 For male patients, the diagnosis needs to be confirmed histologically 
to exclude a malignant lesion and mutations that may be related to future hepatocellular carcinoma (HCC) development. ${ }^{1417-19}$ Management depends mainly on the associated symptoms, lesion size and location. Female patients are advised to stop OC medication and strive for a healthy body weight. ${ }^{49182021}$ Men taking ASs are strongly advised to stop these drugs. Currently, further treatment of (a) symptomatic HCA focuses on an active surveillance policy, aiming to avoid surgery. ${ }^{1418} 1922$ In men, a biopsy is indicated to exclude mutations that may be related to HCC development. ${ }^{14}$ 17-19

For most women management consists of a 6-month surveillance period after which possible regression of the HCA is assessed by MRI. ${ }^{14} 181922$ Additionally, a prediction model may be used to calculate a projected chance of regression to $<5 \mathrm{~cm}$ in 1 or 2 years. ${ }^{21}$ After this initial observation period, management will be based on patient characteristics, size and regression of the HCA, findings on imaging and the predicted chance of regression. Figure 1 shows the strategy from the European Association for the Study of the Liver Clinical Practice Guidelines on the management of benign liver tumours. ${ }^{14} \mathrm{New}$ insights into the mechanism playing a role in adenoma regression are needed to adequately inform patients about the course of follow-up and the necessity of additional interventions in the near future. Faster regression of the HCA could reduce patient's anxiety, waiting time, lead to a more reliable prediction of regression at 1 year and finally, less surgical interventions with its associated risk of complications.

HCA is associated with obesity and metabolic syndrome, ${ }^{10-13}$ anabolic androgen use, ${ }^{23-25}$ rare genetic syndromes including glycogen storage diseases and familial adenomatous polyposis. ${ }^{3} 2627$ HCA consists of several subtypes: inflammatory, steatotic, $\beta$-cateninmutation-associated and unspecified, of which the inflammatory and steatotic subtypes are most prevalent in female subjects. ${ }^{11} 1228$ Subjects with inflammatory HCA have a higher body mass index (BMI) when compared with subjects with steatotic HCA. ${ }^{11}{ }^{12}$ Inflammatory adenomas present more often as multiple lesions, whereas steatotic HCA is more often single lesions. ${ }^{11-13}$ Obese subjects are therefore more likely to have multiple adenomas of the inflammatory subtype. Most cases can be managed by wait and see policy. ${ }^{11-13}$ The mechanism causing the increased risk of developing inflammatory HCA in obese subjects has not been completely identified. ${ }^{11}$ Two mechanisms have been hypothesised: first, adipose tissue accounts for extra glandular formation of oestrogen, which may increase with higher body weight. ${ }^{29} 30$ Patients with obesity have demonstrated higher oestrogen levels when compared with healthy individuals. ${ }^{30-32}$ Weight loss may result in a reduction of sex hormones. ${ }^{33}$ Higher BMI has also been linked with a higher risk of liver tumours, but the biological mechanisms underlying this association have not been clearly identified. ${ }^{31} 32$ A second explanation for the link between overweight and the risk of developing HCA could be persistent inflammation. Enhanced

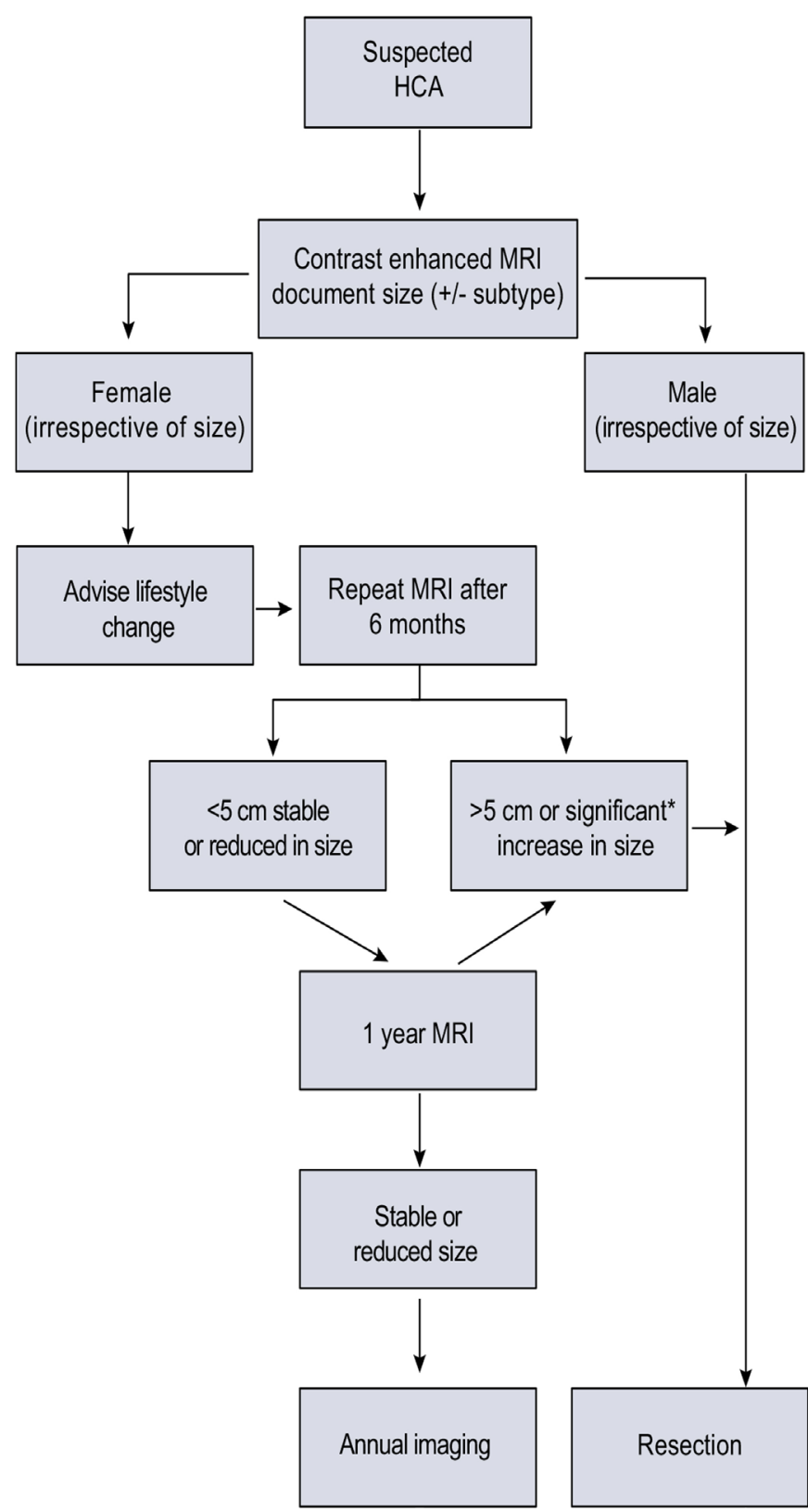

Figure 1 Flow chart helpful in deciding a management strategy for hepatocellular adenoma $(\mathrm{HCA})$, from the European Association for the Study of the Liver Clinical Practice Guidelines on the management of benign liver tumours. A significant increase is defined as an increase $>20 \%$ of the diameter of the HCA.

inflammation is associated with metabolic syndrome, which might promote abnormal cell growth to develop (larger) inflammatory adenomas. ${ }^{11-13} 34$ Reduction of body weight leads to less metabolic-syndrome-associated inflammation. ${ }^{11}{ }^{12}$ Weight loss has initially been suggested and is currently used as adjunct to treatment for HCA, as the HCA may decrease in size and possibly disappear if patients lose weight. ${ }^{12} 16$ It remains to be determined whether this observation is the outcome of weight loss as such, the effect of withdrawal of oestrogen intake, or a combination of both. By introducing a restrictive diet, the effect of the caloric challenge may be unravelled. 
The classic ketogenic diet is a high-fat, adequateprotein and low-carbohydrate diet that produces metabolic changes with similarities to the starvation state, when fat is the main source of energy. ${ }^{35-38}$ Changes in plasma ketones, insulin, glucose, glucagon and free fatty acids can occur within hours of starting the diet and can be profound. ${ }^{35}$ A recent study also shows the beneficial effect of an eucaloric very-low-carbohydrate ketogenic diet on disease control of patients with acromegaly via lowering of insulin-like growth factor (IGF-1) levels. ${ }^{39}$ In a recent randomised controlled trial, patients with type 2 diabetes improved their glycaemic control and lost more weight after being randomised to a low-calorie ketogenic diet (LCKD) and lifestyle online programme rather than a conventional, low-fat diabetes diet online programme. ${ }^{37}$ Potential side effects of the ketogenic diet include constipation, diarrhoea, nausea, vomiting and very rarely pancreatitis, the frequency of which are low. ${ }^{40-42}$ These side effects can lessen with continued diet use and minor adjustments under supervision of a dietician, diet discontinuation is rarely indicated.

Dietary or caloric restriction (DR), meaning reduced intake of food without malnutrition, is associated with extended life span, lower risk of age-associated diseases, improved fitness and increased resistance to acute stress. ${ }^{43-48}$ DR represents a non-invasive, nonexpensive method of acquiring long-term health benefits by reducing caloric intake. ${ }^{48} 49$ The ketogenic diet also leads to spontaneous reduced intake. ${ }^{50} \mathrm{DR}$ and the ketogenic diet share biological responses implicated in metabolite-controlled longevity pathways, via production of ketone bodies. Production of ketone bodies such as $\beta$-hydroxybutyrate from fatty-acid catabolism may operate as endogenous histone deacetylase inhibitors and may contribute to epigenetic control of gene expression, DNA-repair and genome stability. ${ }^{51}$ An LCKD reduces portal insulin concentrations, which downregulate hepatic growth hormone receptors ${ }^{52}$ and reduce IGF-1 synthesis. ${ }^{53}{ }^{54}$ In combination with weight loss due to this diet, it can potentially lead to a reduction in size of an HCA.

To further enhance the active surveillance strategy as a successful treatment modality of HCA, our primary objective is to investigate whether an LCKD increases the rate of regression of HCA in women with overweight. To this end, we designed a single-centre, proof-of-concept interventional matched cohort study.

\section{METHODS AND ANALYSIS}

The proposed study will be conducted at Erasmus University Medical Center Rotterdam, a tertiary academic hospital in the Netherlands.

\section{Study population}

Because of the nature of the disease, only female subjects are eligible for the trial. Our population of interest consists of subjects with an HCA of any size, who are receiving consultation at our outpatient clinic. Our study encompasses two populations: the main population consists of subjects who were recently diagnosed and have a proposed treatment strategy of an initial surveillance period of 6 months, including cessation of OC use. This strategy is decided on after reviewing their case by the multidisciplinary team of experts, including a hepatobiliary surgeon, a hepatologist and a liver radiologist. The second (sub-)population consists of study subjects who already adhered to this initial surveillance period and had no or only minimal $(5 \mathrm{~mm})$ regression of their HCA. Figure 2 shows an overview comparing the moment of inclusion into the study and the runtime of both the main and subpopulation. Inclusion criteria for both populations are age between 18 and 50 years and a BMI $>25 \mathrm{~kg} /$ $\mathrm{m}^{2}$. Patients older than 50 years are most often post menopausal and will not enter a longer follow-up period than 6 months. Combined with the heterogeneity of this (sub) population and the relatively few patients in this (sub) population, we opted to exclude these patients. If a subject meets any of the following exclusion criteria, she will be excluded from participation: current pregnancy or breast feeding, a medical history of diabetes mellitus type 1 or 2 , an insufficient understanding of the Dutch language, inborn errors of metabolism or participation in another clinical intervention study.

\section{Patient and public involvement}

The development of our research question and outcome was informed by experience from our outpatient clinic. Since the current mainstay of treatment of HCA is surveillance, faster regression of the HCA could reduce patient's anxiety, waiting time and imaging procedures. Aiding with weight loss with a potential treatment effect on the size of HCA could reduce anxiety. Patients were not involved in design or recruitment of the study. Results of study will be disseminated to all participants and via the communications department of Erasmus MC to the public.

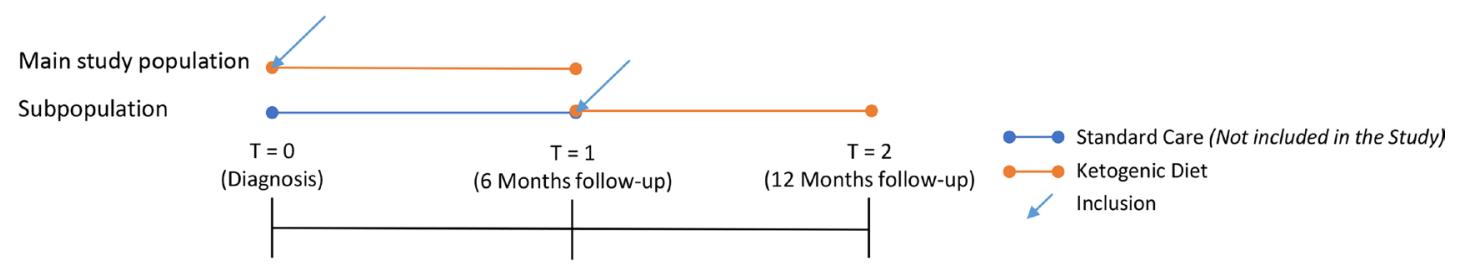

Figure 2 Graphical representation of study runtime for the main study population and the subpopulation. 


\section{Matched historical cohort}

Due to the low incidence of HCA and the proof-of-concept setting, we designed this study as an interventional cohort. To reliably estimate the potential treatment effect of the LCKD on the regression of HCA, we will match our study subjects to a historical control group from a national multicentre cohort. A selection of this large national cohort has been published recently, reporting on the regression of HCAs sized $>5 \mathrm{~cm} .{ }^{21}$ The database constructed from this large multicentre cohort encompasses female patients diagnosed with HCA. Although the published study mainly focused on subjects with an HCA larger than $5 \mathrm{~cm}(\mathrm{n}=180)$, the cohort itself encompasses significantly more subjects $(n>400)$. This also includes subjects with adenomas $<5 \mathrm{~cm}$, thereby being more comparable with our intended study cohort. It also includes the relevant predictors, such as OC use and weight, also investigated in our cohort. Subjects in this cohort were diagnosed and treated according to procedures used in our intended study cohort, aside from adhering to an LCKD diet. Therefore, we estimate that this study population will be a reliable control cohort for our treated study population, after reducing possible influence of covariates by matching. Matching to this earlier acquired cohort will be based on age, use of OCs, weight, type of HCA, size of HCA and number of lesions at baseline. Matching will be conducted via optimal nearest-neighbour matching on propensity score, in a 1:1 ratio without replacement. ${ }^{55}$ This will provide the best available evidence, aside from a randomised controlled trial, since it will replicate a randomised experiment as closely as possible. ${ }^{55}$ Matching will make sure both groups will have a similar distribution of covariates and will therefore reduce the chance of possible bias. With this design, we will be able to include enough study subjects in a relatively short period, and reliably estimate a potential treatment effect. During statistical analysis, regression analysis will be adjusted for possible covariates, as matching and correction for covariates work best in combination. ${ }^{55}$

\section{Intervention}

Participants in the clinical trial will be subjected to an LCKD for 6 months. In this LCKD, the amount of carbohydrates is restricted; in the first 3 months, carbohydrate restriction will be strict, down to $35 \mathrm{~g}$ /day. This amount of carbohydrates will lead to physiological ketosis. In the second part of the study, carbohydrate intake will be increased to $60 \mathrm{~g} /$ day to increase compliance. It is our experience that strict carbohydrate restriction is difficult to maintain in the long term and that liberalising the regime increases compliance. In our earlier study on the effect of a ketogenic diet in patients with acromegaly, ${ }^{39}$ we found that even after increasing carbohydrate intake to $80 \mathrm{~g} /$ day, metabolic improvements were still visible. When adhering to the diet, emphasis is placed on the use of products high in polyunsaturated and monounsaturated fatty acids (diet margarines, oils, fish, nuts), preferably with vegetable and marine sources of protein, in concordance with the national nutritional guidelines. ${ }^{56}$ We aim to moderately reduce the calories to about $70 \%-80 \%$ of the daily energy requirements; therefore, the diet is classified as an LCKD. ${ }^{57}$

\section{Study procedures}

During study runtime, participants will be subjected to several control procedures: online supplemental table 1 and figure 3 provide an overview. The main procedure is defined by regular consultation by a dietician experienced in administering the LCKD. These consultations will focus on explaining the study diet, instructions on how to successfully adhere to the diet and instructions in the case of potential side effects. Patients will receive (person-)tailored advice on acquiring ketosis, are provided information on recipes, certain products they can use and products they should avoid. Nutritional assessment will be conducted to determine dietary intake and thereby also adherence to the LCKD. This assessment will be performed using the 'Eetmeter', ${ }^{58}$ an app extensively used during regular consultations by a dietician. Anthropometric measurements will be collected to assess possible changes in body weight or body composition during the LCKD. Aside from these assessments, subjects will self-check the ketone presence in the urine thrice weekly in the evening, using a simple urine strip to determine ketosis and thereby adherence. Subjects will be carefully instructed on how to use these strips and how to act if they are not in ketosis. During physical study appointments, blood will be acquired by venous puncture for measuring parameters associated with the intervention. After 6 months, a follow-up MRI will be conducted as part of standard of care. The investigator can decide to withdraw a subject from the study for urgent medical reasons, relating to the HCA or the LCKD. Supervision of a qualified dietician is standard in our study and provides close observation to the amount and severity of possible side effects due to the LCKD. Potential side effects can improve with only minor adjustments under supervision of a dietician.

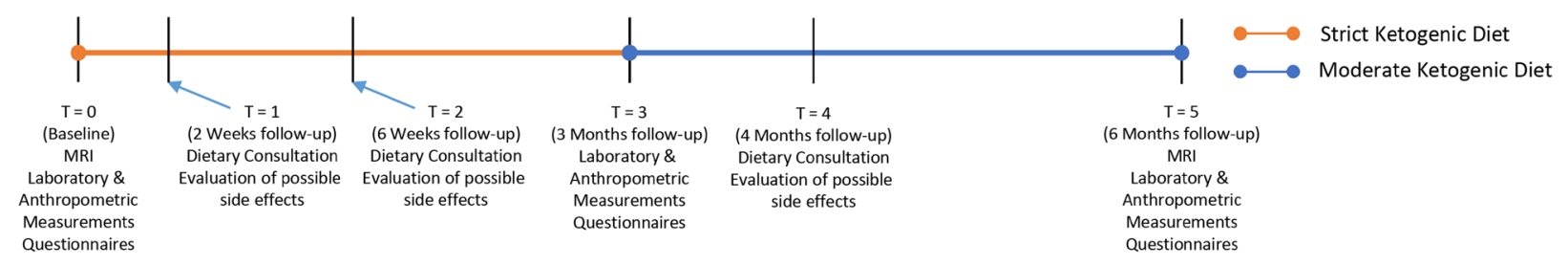

Figure 3 Schematic overview of study runtime and set time points of study procedures. 


\section{Outcomes}

Our primary study endpoint is the regression of HCA and the potential difference in regression when compared with the matched historical controls. The size (largest diameter in $\mathrm{cm}$ ) of the largest HCA diagnosed on MRI will be measured at inclusion and at 6 months after start of the intervention. Mean regression will be calculated after which it will be compared with the mean regression of our matched cohort. The MRI will be conducted according to the standardised protocol for hepatic tumours imaging (Siemens, MRI liver with Primovist, either 3T: HD body array, Aircoil or 1.5T: 48 AA body array, 8Ch cardiac coil, 12Ch Full body array).

Our secondary outcomes comprise several aspects of HCAs and the LCKD:

- Feasibility and adherence to the LCKD will be measured in several ways, including using an internally developed questionnaire which indicates the number of days subjects did not adhere to the ketogenic diet. It will serve as a tool during study visits at the outpatient clinic. Adherence will also be quantified by determining ketone levels in urine, which participants will measure themselves thrice weekly (Siemens, Multi stix 10SG). Dipsticks with results of either,+++ or +++ will be considered as adherent. Patients with non-positive dipstick will be considered as non-adherent. Additionally, blood ketosis will be monitored at the outpatient clinic visits during consultation with the dietician (blood ketone levels of $>0.5 \mathrm{mmol} / \mathrm{L}$ will be considered adherent).

- Side effects and the burden of diet will be assessed via the same internally developed questionnaire during study visits and when a subject contacts the investigators.

- Anthropometric measurements will be performed at the outpatient clinic visits; see online supplemental table 1. These assessments include bodyweight $(\mathrm{kg})$, body composition (Bio-electrical Impedance Analysis with the Bodystat quadscan 4000, Euromedix, Leuven, Belgium) and resting energy expenditure (Quark RMR, Cosmed Benelux B.V., Nieuwegein, The Netherlands).

- Liver fat content will be assessed on the MRI conducted during study runtime, according to the standardised protocol for hepatic adenoma's (Siemens, MRI liver with Primovist, either 3T: HD body array, Aircoil or 1.5T: 48 AA body array, 8Ch cardiac coil, 12Ch Full body array).

- Using the International Physical Activity Questionnaire, ${ }^{59}$ we will assess the physical activity of the participants at set time intervals, a higher score representing more physical activity.

- The RAND-36 questionnaire ${ }^{61-64}$ will be used to assess the quality of life of the participants at set time intervals. A higher score represents a higher quality of life.

- Laboratory measurements, including fasting glucose (mmol/L), fasting insulin (pmol/L), hemoglobin A1c (HbAlc) $(\mathrm{mmol} / \mathrm{mol})$, total cholesterol $(\mathrm{mmol} / \mathrm{L})$, high-density lipoprotein (HDL) (mmol/L), lowdensity lipoprotein (LDL) (mmol/L), free fatty acids $(\mathrm{mmol} / \mathrm{L})$, triglycerides levels $(\mathrm{mmol} / \mathrm{L})$, IGF-1 $(\mathrm{nmol} / \mathrm{L})$, growth hormone $(\mu \mathrm{g} / \mathrm{L})$ and $\beta$-hydroxybutyrate $(\mathrm{mmol} / \mathrm{L})$, will be assessed by routine lab procedures during set time points at the study visits. Measurements will be compared between these time points.

- Nutritional assessment will be measured using the aforementioned Eetmeter ${ }^{58}$ and the internally developed questionnaire. It will provide an overview of macronutrient intake and thereby also adherence. Intake of macronutrients will be compared between set time points.

- Prediction of the probability of HCA regression will be done using the prediction model developed by Klompenhouwer et $a l^{21}$ This prediction model estimates the probability of HCA regression to $<5 \mathrm{~cm}$ at 1 and 2-year follow-up with an internally validated c-index of 0.79. Included predictors in this model are diameter at diagnosis, diameter at first follow-up, the dates of diagnosis and first follow-up and HCAsubtype. It is available via https://hcapredictionshinyappsio/calculator/. The predicted probabilities of regression to $<5 \mathrm{~cm}$ of our cohort of will be calculated and compared with the predicted probabilities of the matched cohort.

In general, if measurements are not available from the matched cohort, change in these measurements will be compared between baseline, at 3 months follow-up and the study endpoint in our interventional cohort. This is, for instance, the case for the quality of life and liver fat content. Follow-up and outpatient visits concerning treatment of the HCA will be identical to standard care. Study appointments will run parallel to standard of care employed and will not interfere with routine procedures.

\section{Sample size calculation}

Although it is a proof-of-concept study, we computed a sample size calculation to support our theorised cohort size. We based our sample size calculation on the large multicentre retrospective cohort mentioned earlier. ${ }^{21}$ This study calculated a median (IQR) HCA diameter at baseline of $82.0 \mathrm{~mm}(65-100)$ and at 6 month follow-up of $65.0 \mathrm{~mm}$ (56-80). Considering a relevant effect of $20 \%$ difference in mean tumour size after 6 months, we estimated an average additional decrease of $13 \mathrm{~mm}$ in the interventional cohort. With a two-sided alpha set at 0.05 , power at 0.95 , we calculated that at least 51 subjects are required. Anticipating a $\pm 10 \%$ dropout, we aim to include 55 participants in this study. We aim to include all study subjects in 1.5-2years, given that our tertiary hospital is a regional referral centre for treatment of HCA. On average, 80 patients with HCA are referred a year. Therefore, we consider it feasible to include all our participants in 2 years, depending on the extent of the COVID-19 pandemic. 


\section{Recruitment}

All eligible subjects visiting the outpatient clinic are invited to participate in the study. The gastroenterologist/hepatobiliary surgeon briefly explains the purpose and the general outline of the study. If potential participants are interested in participation, the study investigator explains the study design, purpose and intervention in more detail. In case informed consent is given, subjects will receive an appointment with the study researcher and the dietician-researcher to start with the LCKD.

\section{Data collection methods}

Study data will be collected from the electronic health system. Questionnaires can either be submitted digitally or on paper, depending on the preference of the participant. Data retrieval will be conducted by trained medical personnel with knowledge of the study procedures. Each study subject has a personal electronical Case Report Form, of which a blank copy can be supplied on request. As described earlier, subjects will be carefully instructed by dieticians, which is why we expect a relatively high participant retention and complete follow-up. If a subject aims to discontinue participation, we ask permission to collect the information from the standard of care MRI at 6 months. Retrieved study data will be handled confidentially and stored in Castor EDC (Amsterdam, the Netherlands), used in compliance with laws and regulations. Data entry will be conducted by trained medical personnel and data will be range-checked during entry to further enhance data quality.

\section{Statistical analysis}

Statistics will be computed using IBM SPSS software V.25.0 or R V.4.0.3 or newer. An intention-to-treat analysis will be conducted to account for the feasibility of the diet. A two-sided significance level of 0.05 will be used for all primary and secondary analyses unless stated otherwise. In the case of missing data, an analysis will be performed with multiple imputation.

To determine the primary endpoint, we will construct a multivariable linear regression model to estimate the difference in tumour regression between the treatment group and the historical control group. The model will be adjusted for age, change in body weight, the ketogenic diet and OC use at baseline. In the secondary analysis, the multivariable linear regression model analysis from the primary analysis will be performed including adherence to the diet as independent variable.

Analysis for adherence to and side effects of the ketogenic diet will be done via descriptive statistics. Change in liver fat content will be compared in our acquired cohort by a multivariable linear regression model. The independent variables in this model will include age, change in body weight and OC use at baseline. An additional analysis will be performed including adherence to the diet as independent variable. Changes in laboratory measurements will be compared in our cohort by using descriptive statistics, t-tests in the case of continuous outcome variables with normal distribution or non-parametric tests in the case of non-normality. The $\chi^{2}$ test will be used for categorical data. Because of repeated measurements, change in body weight, body composition and resting energy expenditure will be estimated in our cohort by mixed-effect models. The independent variable in this model will include age at baseline; an additional analysis will be performed including adherence to the diet as independent variable. Change in quality of life will be compared over time in our cohort by a mixed-effects model analysis. The independent variables in this model will include age and change in body weight. An additional analysis will be performed including adherence to the diet as independent variable. Change in physical activity will also be compared by a mixed-effect model analysis. The independent variables in this model will include age and compliance to the diet. Results of the prediction model will be compared between our cohort and the matched historical controls by the $\chi^{2}$ test.

\section{Data monitoring}

We classified the risk of our study as negligible. As described by the Erasmus MC monitoring plan, we will have a monitoring frequency of once per year. Monitoring activities are as follows: confirming that the Trial Master File and Investigator Site File are present and complete, confirming that the study staff is adequately instructed on the study procedures, assessment of patient inclusion rate, consent, compliance, source document verification and verification of accessibility of study procedures. The frequency and procedures of auditing will not be changed for our study. Regular auditing, standard for this academic hospital, will be continue as normal. This process is done independently from the study and its investigators.

Conservative treatment of (a)symptomatic HCA in women of fertile age has been proven feasible and safe and is current best clinical practice. ${ }^{18} 192122$ Especially asymptomatic adenoma's $<5 \mathrm{~cm}$ have an extremely low chance of rupture ${ }^{6566}$ and are treated almost exclusively by careful follow-up. Adenomas larger than $5 \mathrm{~cm}$ in subjects who are using OCs at the time of diagnosis also have a significant chance of an excellent response on conservative therapy. ${ }^{18} 192122$ Subjects will receive adequate information considering the nature of the disease and will be carefully instructed when to contact the hospital and/ or their general practitioner. Conservative treatment is a low-risk therapy of HCA and continuing this practice in the current study does not increase the risk of clinically significant complications. Potential side effects of the ketogenic diet are constipation, diarrhoea, nausea, vomiting and very rarely pancreatitis, the frequency of which are low. ${ }^{40-42}$ Supervision of a qualified dietician during study runtime will adequately detect potential side effects. Possible side effects will often improve with minor adjustments after dietary consultation. Therefore, we consider it safe to use a ketogenic diet for our subjects 
and the risks associated with participation can be considered negligible.

\section{ETHICS AND DISSEMINATION}

The medical ethical committee of Erasmus MC has approved the study protocol, patient information files, consent procedures and other study-related documents and procedures. This medical committee is an extension of the Dutch Central Committee on Research Involving Human Subjects. The trial has been registered under medical ethical assessment numbers MEC-2020-0777 and NL75014.078.20.

The procedure regarding study amendments has been defined in the study protocol. A substantial amendment is defined as an amendment that is likely to affect to a significant degree: the safety or physical or mental integrity of the subjects of the trial, the scientific value of the trial, the conduct or management of the trial or the quality or safety of any intervention used in the trial. Substantial amendments will be submitted to the same medical ethical committee and will only be conducted and implemented after approval. If an amendment has been accepted by the ethical committee, it will be changed accordingly in the trial registry and any previous publications.

Informed consent will be obtained in accordance with relevant laws and regulations (eg, Guideline for Good Clinical Practice (ICH-GCP)) by the qualified study researchers or principal investigator. Because our research will not be performed in minors or incapacitated subjects, no information file or procedure has been defined for surrogates. A separate additional consent has been constructed for the use of participant data for related studies in the future; this consent is not required to participate in the study. A model consent form has been provided in the online supplemental appendices.

\section{Confidentiality}

On study inclusion, each subject will be assigned a study number which will be listed on all study related documentation. In this study, no personal documents will be listed. Data will be confidentially saved for a maximum of 15 years after completion of the study, in compliance with Dutch practice and laws. All personal or pseudonymised data will be handled with care and in compliance with the EU General Data Protection Regulation. Data entry will be conducted in the pseudonymised form with study numbers. The principal investigator will keep a subject identification log; a record of the personal identification data linked to each study number. This record is filed at the investigational site and can only be accessed by authorised personnel, f.i. the investigator and the supporting site staff. Research data that can be traced to individual persons can only be viewed by authorised personnel. The principal investigator is responsible for final trial data set. Research data can only be viewed by authorised personnel: members of the research team, members of the healthcare inspection and study monitors. Potential data sharing requests will be approved by a representative of the academic hospital and the principal investigators.

After the study period of 6 months, participants are free to continue the LCKD: they can choose to keep adhering or switch to another diet. If they would like advice from a dietician, they can be referred to a dietician via their primary care physician. Potential compensation for any participant who suffers harm due to trial participation has been covered by the academic hospital insurance, in accordance with laws and regulations.

\section{Dissemination of study findings}

The principal investigator is responsible for the public disclosure and publication of the research data. The (final) results of the study will be summarised in a report/ article and will be submitted for publication in a medical journal, adhering to the Strengthening the Reporting of Observational Studies in Epidemiology checklist for cohort studies. The trial has also been registered in a Dutch public trial registration registry, available at: http://www. trialregister.nl/trialreg/index.asp. Eligibility for authorship has been defined by the International Committee of Medical Journal Editors (ICMJE) guidelines for the role of Authors and Contributors. ${ }^{67}$ The full protocol, anonymised dataset and statistical code can be granted after submitting a request with the principal investigator and the institutions technology transfer office.

The primary objective of our study is to investigate whether an LCKD increases the rate of regression of HCA in overweight women. To this end, we designed an interventional matched cohort study. Our study has several limitations. First, our study is not designed as a randomised controlled trial. Due to the low incidence of HCA, it is unfeasible to construct an ethically and scientifically sound randomised controlled trial, which could be completed in a timely manner. Therefore, we designed a matched cohort intervention study, creating the opportunity to provide the best available scientific evidence on the therapeutic effect of an LCKD on HCA, currently a knowledge gap in this research field. We have a large national cohort at our disposal, ${ }^{21}$ which is a reliable control cohort for our treated study population, after reducing possible influence of covariates by matching. This will provide the best available evidence, aside from a randomised controlled trial.

Although we have a relatively large and highly motivated population of patients with HCA, another limitation of our study is that the dietary intervention is at risk for unforeseen non-adherence due to the long intervention period. We aim to reduce this risk by providing comprehensive information and assistance during their LCKD.

\section{Trial status}

This trial is currently ongoing and actively recruiting participants $(14 / 55)$. It has a planned duration of 2 years, with recruitment having started in April 2021 and continuing to April 2023. All changes in the study protocol will be recorded in the clinical trials registry. 


\section{Author affiliations}

${ }^{1}$ Erasmus MC Transplant Institute, Department of Surgery, Division of Hepatobiliary and Transplantation Surgery, Erasmus MC, Rotterdam, Zuid-Holland, The Netherlands

${ }^{2}$ Princess Maxima Center for Pediatric Oncology, Utrecht, The Netherlands ${ }^{3}$ Department of Internal Medicine, Division of Dietetics, Erasmus MC, Rotterdam, Zuid-Holland, The Netherlands

${ }^{4}$ Department of Hepato-Gastroenterology, Erasmus MC, Rotterdam, Zuid-Holland, The Netherlands

${ }^{5}$ Department of Internal Medicine, Division of Endocrinology, Erasmus MC, Rotterdam, Zuid-Holland, The Netherlands

${ }^{6}$ Erasmus MC Cancer Institute, Department of Molecular Genetics, Erasmus MC, Rotterdam, Zuid-Holland, The Netherlands

Acknowledgements The authors would like to thank F. de Lange for her contributions to the study protocol and study documents and their expertise regarding the medical ethical submittance proces. We additionally would like to thank R.C.A van Linschoten for his epidemiological expertise and statistical consultation.

Contributors All authors have been extensively involved in study design. Specifically, forming the hypothesis, designing the study and designing and formulating the study intervention was conducted by JI, CAJO, KAB, EJTMvdL and RdM. JHJH and A-JvdL supported the study design via an advisory role for both the intervention, measurements and the methodological quality of the study. During study runtime, the inclusion of potential study subjects will be performed by CAJO, $\mathrm{KAB}$ and EJTMvdL. The study intervention will be conducted by KAB and EJTMvdL. The collection, management, analysis and interpretation of data will be performed by CAJO. The writing of the report and decision to submit will be made by all authors.

Funding The design and implementation of this study was conducted without support of a specific grant from any funding agency in the public, commercial or not-for-profit sectors. Research and writing of this manuscript were indirectly supported by ONCODE (Dutch Cancer Society), but ONCODE had no part in formulating the hypothesis, construction of the study design or conduction of the trial. JHJH was additionally supported by the European Research Council Advanced Grant Dam2Age, and NIH grant (P01 AG017242), as well as BBoL (NW0-ENW) and the Deutsche Forschungsgemeinschaft - Project-ID 73111208 - SFB 829.

Competing interests None declared.

Patient consent for publication Consent obtained directly from patient(s)

Provenance and peer review Not commissioned; externally peer reviewed.

Supplemental material This content has been supplied by the author(s). It has not been vetted by BMJ Publishing Group Limited (BMJ) and may not have been peer-reviewed. Any opinions or recommendations discussed are solely those of the author(s) and are not endorsed by BMJ. BMJ disclaims all liability and responsibility arising from any reliance placed on the content. Where the content includes any translated material, BMJ does not warrant the accuracy and reliability of the translations (including but not limited to local regulations, clinical guidelines, terminology, drug names and drug dosages), and is not responsible for any error and/or omissions arising from translation and adaptation or otherwise.

Open access This is an open access article distributed in accordance with the Creative Commons Attribution Non Commercial (CC BY-NC 4.0) license, which permits others to distribute, remix, adapt, build upon this work non-commercially, and license their derivative works on different terms, provided the original work is properly cited, appropriate credit is given, any changes made indicated, and the use is non-commercial. See: http://creativecommons.org/licenses/by-nc/4.0/.

\section{ORCID iD}

Christiaan Albert Johan Oudmaijer http://orcid.org/0000-0003-4852-6514

\section{REFERENCES}

1 Rooks JB, Ory HW, Ishak KG, et al. Epidemiology of hepatocellular adenoma. The role of oral contraceptive use. JAMA 1979;242:644-8.

2 Reddy KR, Schiff ER. Approach to a liver mass. Semin Liver Dis 1993;13:423-35

3 Leese T, Farges O, Bismuth $\mathrm{H}$. Liver cell adenomas. A 12-year surgical experience from a specialist hepato-biliary unit. Ann Surg 1988;208:558-64.
4 Edmondson HA, Reynolds TB, Henderson B, et al. Regression of liver cell adenomas associated with oral contraceptives. Ann Intern Med 1977;86:180-2.

5 Edmondson HA, Henderson B, Benton B. Liver-Cell adenomas associated with use of oral contraceptives. $N$ Engl J Med 1976;294:470-2.

6 Giannitrapani L, Soresi M, La Spada E, et al. Sex hormones and risk of liver tumor. Ann N Y Acad Sci 2006;1089:228-36.

7 Rosenberg L. The risk of liver neoplasia in relation to combined oral contraceptive use. Contraception 1991;43:643-52.

8 Kawakatsu M, Vilgrain V, Erlinger S, et al. Disappearance of liver cell adenoma: CT and MR imaging. Abdom Imaging 1997;22:274-6.

9 Aseni P, Sansalone CV, Sammartino C, et al. Rapid disappearance of hepatic adenoma after contraceptive withdrawal. J Clin Gastroenterol 2001;33:234-6.

10 Chang CY, Hernandez-Prera JC, Roayaie S, et al. Changing epidemiology of hepatocellular adenoma in the United States: review of the literature. Int J Hepatol 2013;2013:1-7.

11 Bioulac-Sage P, Taouji S, Possenti L, et al. Hepatocellular adenoma subtypes: the impact of overweight and obesity. Liver Int 2012;32:1217-21.

12 Bröker MEE, Gaspersz MP, Klompenhouwer AJ, et al. Inflammatory and multiple hepatocellular adenoma are associated with a higher BMI. Eur J Gastroenterol Hepatol 2017;29:1183-8.

13 Bunchorntavakul C, Bahirwani R, Drazek D, et al. Clinical features and natural history of hepatocellular adenomas: the impact of obesity. Aliment Pharmacol Ther 2011;34:664-74.

14 European Association for the Study of the Liver (EASL). EASL clinical practice guidelines on the management of benign liver tumours. $J$ Hepatol 2016;65:386-98.

15 Bonder A, Afdhal N. Evaluation of liver lesions. Clin Liver Dis 2012;16:271-83.

16 Dokmak S, Belghiti J. Will weight loss become a future treatment of hepatocellular adenoma in obese patients? Liver Int 2015;35:2228-32.

17 Agrawal S, Agarwal S, Arnason T, et al. Management of hepatocellular adenoma: recent advances. Clin Gastroenterol Hepatol 2015;13:1221-30.

18 van Aalten SM, Terkivatan T, de Man RA, et al. Diagnosis and treatment of hepatocellular adenoma in the Netherlands: similarities and differences. Dig Surg 2010;27:61-7.

19 van Aalten SM, Witjes CDM, de Man RA, et al. Can a decisionmaking model be justified in the management of hepatocellular adenoma? Liver Int 2012;32:28-37.

20 Ault GT, Wren SM, Ralls PW, et al. Selective management of hepatic adenomas. Am Surg 1996;62:825-9.

21 Klompenhouwer AJ, Alblas M, van Rosmalen BV, et al. Development and validation of a model to predict regression of large size hepatocellular adenoma. Am J Gastroenterol 2019;114:1292-8.

22 van Aalten SM, Verheij J, Terkivatan T, et al. Validation of a liver adenoma classification system in a tertiary referral centre: implications for clinical practice. $J$ Hepatol 2011;55:120-5.

23 Nakao A, Sakagami K, Nakata Y, et al. Multiple hepatic adenomas caused by long-term administration of androgenic steroids for aplastic anemia in association with familial adenomatous polyposis. $J$ Gastroenterol 2000;35:557-62.

24 Resnick MB, Kozakewich HP, Perez-Atayde AR. Hepatic adenoma in the pediatric age group. clinicopathological observations and assessment of cell proliferative activity. Am J Surg Pathol 1995; 19:1181-90.

25 Velazquez I, Alter BP. Androgens and liver tumors: Fanconi's anemia and non-Fanconi's conditions. Am J Hematol 2004;77:257-67.

26 Labrune P, Trioche P, Duvaltier I, et al. Hepatocellular adenomas in glycogen storage disease type I and III: a series of 43 patients and review of the literature. J Pediatr Gastroenterol Nutr 1997;24:276-9.

27 Talente GM, Coleman RA, Alter C, et al. Glycogen storage disease in adults. Ann Intern Med 1994;120:218-26.

28 Bioulac-Sage P, Laumonier H, Couchy G, et al. Hepatocellular adenoma management and phenotypic classification: the Bordeaux experience. Hepatology 2009;50:481-9.

29 Gruber CJ, Tschugguel W, Schneeberger C, et al. Production and actions of estrogens. N Engl J Med 2002;346:340-52.

30 Nelson LR, Bulun SE. Estrogen production and action. J Am Acad Dermatol 2001:45:S116-24

31 Rui R, Lou J, Zou L, et al. Excess body mass index and risk of liver cancer: a nonlinear dose-response meta-analysis of prospective studies. PLoS One 2012;7:e44522.

32 Wang $\mathrm{Y}$, Wang B, Shen F, et al. Body mass index and risk of primary liver cancer: a meta-analysis of prospective studies. Oncologist 2012:17:1461-8. 
33 Rock CL, Pande C, Flatt SW, et al. Favorable changes in serum estrogens and other biologic factors after weight loss in breast cancer survivors who are overweight or obese. Clin Breast Cancer 2013;13:188-95.

34 Paradis V, Champault A, Ronot $\mathrm{M}$, et al. Telangiectatic adenoma: an entity associated with increased body mass index and inflammation. Hepatology 2007;46:140-6.

35 Yancy WS, Westman EC, McDuffie JR, et al. A randomized trial of a low-carbohydrate diet vs orlistat plus a low-fat diet for weight loss. Arch Intern Med 2010;170:136-45.

36 Walczyk T, Wick JY. The ketogenic diet: making a comeback. Consult Pharm 2017;32:388-96.

37 Saslow LR, Mason AE, Kim S, et al. An online intervention comparing a very low-carbohydrate ketogenic diet and lifestyle recommendations versus a plate method diet in overweight individuals with type 2 diabetes: a randomized controlled trial. $\mathrm{J}$ Med Internet Res 2017;19:e36.

38 Hartman AL, Vining EPG. Clinical aspects of the ketogenic diet. Epilepsia 2007;48:31-42.

39 Coopmans EC, Berk KAC, El-Sayed N, et al. Eucaloric Very-LowCarbohydrate ketogenic diet in acromegaly treatment. $N$ Engl $\mathrm{J} \mathrm{Med}$ 2020;382:2161-2.

40 McDonald TJW, Cervenka MC. Ketogenic diets for adult neurological disorders. Neurotherapeutics 2018;15:1018-31.

41 Westman EC, Feinman RD, Mavropoulos JC, et al. LowCarbohydrate nutrition and metabolism. Am J Clin Nutr 2007;86:276-84

42 Feinman RD, Pogozelski WK, Astrup A, et al. Dietary carbohydrate restriction as the first approach in diabetes management: critical review and evidence base. Nutrition 2015;31:1-13

43 Colman RJ, Anderson RM, Johnson SC, et al. Caloric restriction delays disease onset and mortality in rhesus monkeys. Science 2009;325:201-4.

44 Cowen T, Johnson RJ, Soubeyre V, et al. Restricted diet rescues rat enteric motor neurones from age related cell death. Gut 2000;47:653-60.

45 Levolger S, van den Engel S, Ambagtsheer G, et al. Caloric restriction is associated with preservation of muscle strength in experimental cancer cachexia. Aging 2018;10:4213-23.

46 Nakagawa S, Lagisz M, Hector KL, et al. Comparative and metaanalytic insights into life extension via dietary restriction. Aging Cell 2012;11:401-9.

47 Robertson LT, Mitchell JR. Benefits of short-term dietary restriction in mammals. Exp Gerontol 2013;48:1043-8.

48 Willcox DC, Willcox BJ, Todoriki $\mathrm{H}$, et al. Caloric restriction and human longevity: what can we learn from the Okinawans? Biogerontology 2006;7:173-7.

49 Redman LM, Heilbronn LK, Martin CK, et al. Effect of calorie restriction with or without exercise on body composition and fat distribution. J Clin Endocrinol Metab 2007:92:865-72.
50 Gibson AA, Seimon RV, Lee CMY, et al. Do ketogenic diets really suppress appetite? A systematic review and meta-analysis. Obes Rev 2015;16:64-76.

51 Di Francesco A, Di Germanio C, Bernier M, et al. A time to fast. Science 2018:362:770-5.

52 Leung KC, Doyle N, Ballesteros M, et al. Insulin regulation of human hepatic growth hormone receptors: divergent effects on biosynthesis and surface translocation. J Clin Endocrinol Metab 2000;85:4712-20.

53 Ho PJ, Friberg RD, Barkan AL. Regulation of pulsatile growth hormone secretion by fasting in normal subjects and patients with acromegaly. J Clin Endocrinol Metab 1992;75:812-9.

54 Clemmons DR. Involvement of insulin-like growth factor-I in the control of glucose homeostasis. Curr Opin Pharmacol 2006;6:620-5.

55 Stuart EA. Matching methods for causal inference: a review and a look forward. Stat Sci 2010;25:1-21.

56 Kromhout D, Spaaij CJK, de Goede J, et al. The 2015 Dutch foodbased dietary guidelines. Eur J Clin Nutr 2016;70:869-78.

57 Trimboli P, Castellana M, Bellido D, et al. Confusion in the nomenclature of ketogenic diets blurs evidence. Rev Endocr Metab Disord 2020;21:1-3.

58 "Mijn Eetmeter". Available: https://mijn.voedingscentrum.nl/nl/ eetmeter/ [Accessed 09-02-2022].

59 International physical activity questionnaire. Beschikbaar via. Available: www.ipaq.ki.se

60 Gosselink R. KNGF-richtliijn COPD. Amersfoort: Koninklijk Nederlands Genootschap voor Fysiotherapie, 2008. www. kngfrichtlijnen.nl

61 Rand: Ware JE, Sherbourne Cd (1990); Nederlandse versie: SF-36 Aaronson N 1998.

62 der ZKIvan, Sanderman R. Het meten van de algemene gezondheidstoestand Met de RAND-36, Een handleiding. Tweede herziene druk. UMCG / Rijksuniversiteit Groningen, research Institute share, 2012.

63 Aaronson NK, Muller M, Cohen PD, et al. Translation, validation, and norming of the Dutch language version of the SF-36 health survey in community and chronic disease populations. J Clin Epidemiol 1998:51:1055-68.

64 VanderZee KI, Sanderman R, Heyink JW, et al. Psychometric qualities of the RAND 36-Item Health Survey 1.0: a multidimensional measure of general health status. Int J Behav Med 1996;3:104-22.

65 Bieze M, Phoa SSKS, Verheij J, et al. Risk factors for bleeding in hepatocellular adenoma. Br J Surg 2014;101:847-55.

66 Deneve JL, Pawlik TM, Cunningham S, et al. Liver cell adenoma: a multicenter analysis of risk factors for rupture and malignancy. Ann Surg Oncol 2009;16:640-8.

67 Defining the role of authorsInternational Committee of Medical Journal Editors. Available: http://www.icmje.org/recommendations/ browse/roles-and-responsibilities/defining-the-role-of-authors-andcontributors.html [Accessed 09-02-2022]. 